\title{
Somebody, Sometime, Somewhere, Something
}

\author{
Stefano Spaccapietra, Lina Al-Jadir, Shijun Yu \\ École Polytechnique Fédérale de Lausanne, EPFL \\ \{stefano.spaccapietra,lina.al-jadir,shijun.yu\}@epfl.ch
}

\begin{abstract}
The success of the Web and of portable devices has made popular the idea that any information could be made available to anybody, anytime, and anywhere. Other paradigms are taking off within the ubiquitous computing solutions. This invited paper identifies some of these paradigms, focusing on the more innovative ones in terms of information services. In particular, broadcast-based and location-based services are introduced. Further, it discusses user profiles and contexts, two essential components for modern services.
\end{abstract}

\section{Introduction 1}

"Ubiquitous computing technologies provide a pervasive base for a real world environment such that we can acquire and deliver information at every place. Advances in the technologies of displays, electronic papers, digital architecture, sensors, RFID tags and storage devices etc. may bring a new real world environment for data access." These first two sentences from the call for papers of the International Workshop on Ubiquitous Data Management (UDM'05) describe the background scene that supports a variety of new approaches to data management and dissemination and that is enabled by wireless communication.

Ubiquitous computing and ubiquitous data management may be seen as generic terms covering a large spectrum of facilities. Some of them just reproduce services that we are used to have in traditional, centralized systems. This is the case, in particular, of the facilities aiming at allowing people on the move to continue doing business as usual, irrespectively of their

\footnotetext{
${ }^{1}$ The ideas presented in this paper rely on work that was partly carried out in the framework of the MICS NCCR, funded by Swiss FNRS under grant number 5005-67322, and in the framework of the EPFL Center for Global Computing, supported by the Swiss National Funding Agency OFES as part of the European projects KnowledgeWeb (FP6-507482) and DIP (Data, Information, and Process Integration with Semantic Web Services) No 507483.
}

current location and of current time. The explicitly stated target is to give access to any information, to anybody, anywhere and anytime. While this may give the impression that we live in a pervasive information space, the services offered to users show little advances in terms of information management techniques, the major novelty residing in the underlying mobile communication systems.

More innovative approaches to information services exist. They aim at avoiding the major and wellknown drawbacks of the current situation, which sees users in search of useful information flooded by huge amounts of poorly relevant data. We believe such approaches are promised to a brilliant future, as personalized information services, rather than business-oriented services, are the emerging trend that nowadays boosts IT development.

Search for ad-hoc information is indeed the typical activity of individuals on the move, complementing other major communication-oriented activities such as exchanging messages (now including images) via email, SMS, or any other communication channel. Thus, on-the-spot information delivery appears as the new challenge for information services. This includes the need to provide information tailored for the specific requesting user and taking into account contextual factors influencing the user perception of what is relevant information.

This paper is an introductory exploration of the kind of services that can be designed to cope with the new requirements for on-the-spot information delivery. It considers broadcasting-based services, locationbased services, and pervasive services.

\section{On-the-spot information delivery}

We are interested here in how information can be delivered to users preferably without resorting to querying a central information service.

The first contribution to the goal relies on wireless communication and consists in the facility to deliver information wherever the user is. This comes with a number of important technical issues about humancomputer interaction (HCI), i.e., what is the best way 
to communicate with users taking into account the constraints imposed by the limited functionality of the communication device (e.g., cellular phone, PDA). Multimodal interfaces, e.g., using speech recognition and speech generation, are part of the answer to the problem. We do not discuss HCI issues any further, as we do not see these as specifically related to information services.

More relevant to this paper are issues about determining what information has to be delivered, and when. Simply stated, the common aim is to deliver information when it may be useful, and information that is relevant to what the user is currently doing and to the targeted user. Examples of inappropriate timing include delivering the requested information too late (e.g., providing details about the next train the user may catch after the departure of the train), delivering information that is currently useless (e.g., information on services offered by a bank whose offices are closed for the next three days), and providing "wrong" information (e.g., weekdays travel schedule on Saturdays or Sundays).

Regarding relevance to the targeted users, obviously a major goal for personalized services, it can be achieved only if appropriate information about the user is available to the service provider. Such information is usually referred to as the "user profile". Acquiring and maintaining useful user profiles is an uneasy task. It includes managing several profiles for the same user, in an attempt to tailor the processing of an information request to the current interests of the user, likely to depend on his/her activity (what (s)he is doing).

Another well-known input towards increasing relevance of the returned information is taking into account contextual information not attached to the individual user (for this, we have the user profiles) but to his/her actual environment, i.e. data that has to do with where the user is located and when the request is issued, and that may have an influence on the information retrieval strategy or on information selection. More on this follows in section 6 .

While user profiles and context management are inherent aspects of personalized information services, whatever the chosen approach is, the way information is gathered and disseminated determines alternative service architectures and solutions. Automatic and anonymous dissemination is achieved by broadcastbased services, while on request and locally focused dissemination characterizes location-based services.

\section{Broadcast-based Services}

Information broadcasting denotes the communication technique where emitting sources disseminate in- formation to the surrounding world, without targeting a specific individual receiver. Receivers are all devices located within the range of the emission and which have the capability to capture the information.

In the pervasive information space that ubiquitous computing enables, we can easily foresee a huge amount of information sources with a very limited emission range (e.g., a few meters). Industry is likely to be able to produce thousands of miniaturized information broadcast devices at minimal cost, which makes it possible to imagine that, for instance, each shop is equipped with such a device. Each source would broadcast its current message, which can thus be captured by passers-by. The intention is to enable scenarios alike the following one:

- Chieko is walking in the street, she keeps her cellular phone on;

- She walks by a convenience store; her phone receives a call from the store to prompt her with special offers. Her cellular checks if offers correspond to her profile. If something matches her interests, the phone rings;

- She walks by a fashion store. The phone gets the message from the store but discards it as the store targets teenagers;

- She walks by a bank, but the bank is closed. No message is sent/received.

In this scenario we assumed the cellular phone has the ability to filter incoming messages, based on the user profile. Alternatively, we can also imagine that the emitting source only activates its message broadcasting when some user with a corresponding user profile is in the vicinity.

The information dissemination paradigm behind our broadcasting scenario is the one that aims at delivering some specific information (e.g., the advertisement message from the shop) to some specific users (those in the vicinity, because these are the users that may immediately react to the message, e.g. decide to enter the shop), at some appropriate time (e.g., only while the shop is open) and within a given area (the emission range). In short, we can call it the $4 \mathrm{~S}$ paradigm: Somebody, Sometime, Somewhere, Something. Push location-based services [11] pursue a similar idea.

We believe this paradigm is an interesting complement to the current 4A paradigm (Anybody, Anytime, Anywhere, Anything). Unfortunately, it is getting too little attention from industry and academia. Think, for example, of the audio devices that are being distributed to museum visitors so that they can get information on works on display. With current technology, visitors are requested to type the identifier of the work to get the corresponding information. As usual with similar devices, many visitors experience problems in finding the 
way to correctly dial the number and to control the flow of information (e.g., stop, reactivate). The broadcast approach would see a broadcasting chip installed next to each work, and visitors would just need to be in front of the work to receive the information. No manipulation required anymore, just moving around.

\section{Location-based Services}

In contrast to broadcasting services, LocationBased Services (LBS) have become an increasingly popular approach, and academia as well as industry is heavily investing in their development. LBS [8] aim at providing users with useful, local information, i.e., information that belongs to a particular domain of interest to the user and can be of use while the user remains in the area where (s)he currently is. The most popular example of information provided by LBS is "find the nearest restaurant". Fortunately, LBS can be designed to provide more elaborate information, in particular by taking into account the user's profile and other contextual data. For example, LBS can implement sophisticated mobile marketing techniques based on detailed knowledge of customer profiles, history, needs, and preferences [2]. This implies techniques able to integrate location information, customer needs, and vendor offerings.

Real LBS use the decentralized knowledge that is geographically close to the user's location and is consequently most likely to hold the information the moving user wants to retrieve in order to decide about his/her behavior in the short term (within the next hours or days). Tourist assistance, traffic monitoring, and mobile office are frequently quoted examples of LBS applications. Most proposals consider pull-style services (reacting to user's requests, e.g. find a restaurant), rather than the above-mentioned push-style services [11]. Also, most LBS are thought of as information-providing services, while agent-like services, capable of performing an action, could be provided as well (e.g., a service to book a seat for the concert at the local opera house tonight). A typical example scenario for LBS is:

- A person is walking in the city with a PDA, the city has some attractions. When approaching a church, the person asks for information about its history and characteristics. At lunchtime, the person asks for nearby restaurants and their menus.

While this scenario resembles the one we described for broadcast-based services, there are major differences. In particular, in LBS information is gathered from one or more local sources, usually in response to users' requests (pull style), it does not come unsolicited (push) from a collection of independently acting sources.

What contributes in making LBS different from Web information services is their intentionally limited geographical scope (their knowledge-base only covers a limited region), on the one hand, and, on the other hand, the fact that they can basically be seen as mediators between a generally unknown, usually mobile, user and data sources or service providers that may have to be dynamically discovered.

Under this assumption, LBS not only need user profiles and contextual data to personalize the information retrieval; they also need what we call a "data profile", i.e. a description of what kind of data they may find in the locally and currently available data sources. These data profiles enable LBS to find out where information of interest for the query on hand may be expected.

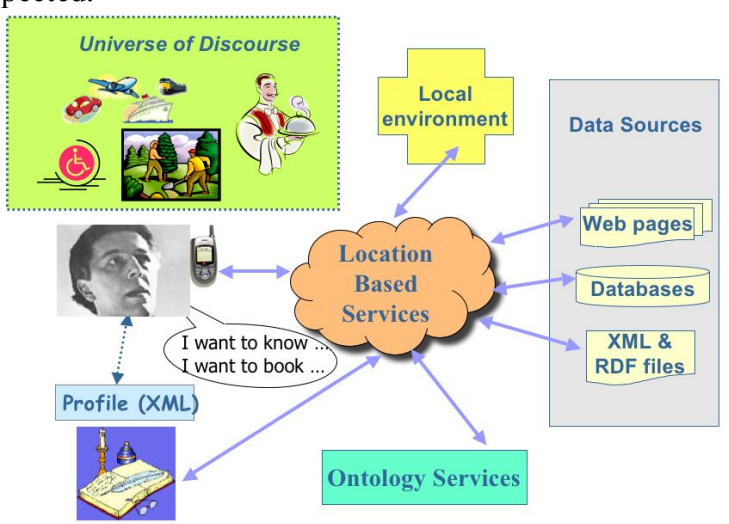

Figure 1. An LBS framework.

Figure 1 shows an architectural framework for LBS. It includes a generic data source about the local environment, providing contextual information (e.g., atmospheric conditions, traffic intensity) that may influence user preferences and behavior. Local data sources include Web pages with local information (e.g., Web pages from the local tourist office). The framework also includes an ontological source providing help to understand how user profiles and context data can be matched to data profiles. LBS can be designed as generic services or as localized services. The former sees LBS as, for example, a Web service that has no associated data sources. Users may call it to get information related to their geographical vicinity. The actual GPS position of the user would be the input that the LBS uses to search for local data. Once local data sources are found and connected, the LBS can start responding to requests for information. On the other hand, a localized LBS is already tailored to a specific geographical region, i.e. it has an associated set of known data sources and can start processing user que- 
ries as soon as they are received. The advantage of localized LBS is that they are immediately operational, while generic LBS need a setting up phase to acquire the data. Moreover, localized LBS can be tailored to local peculiarities, which would make them more effective than generic LBS. Conversely, the advantage of generic LBS is that they can maintain history of user interactions (assuming the same user repeatedly calls the same LBS) and can therefore more easily adjust the search for information to user's preferences, thus increasing relevance of the returned information. However, counterparts to this advantage are privacy threats, and knowledge about user location and trajectories needs to be protected.

From a semantic viewpoint, the major characteristic of, and challenge for, LBS is the fact that their mediation between users and data sources has to be run onthe-fly, i.e., it cannot be prepared in advance, as the partners in the mediation are not necessarily known. We could say that LBS have to perform query evaluation in a hostile environment. To overcome the difficulty, contributions from most advanced techniques are welcome. They include: ontology assistance (to understand what it is all about), peer-to-peer information search (to increase chances of finding relevant information), incomplete information handling (to cope with missing data), and approximation techniques (to determine what could be a reasonable answer when a perfect matching is not possible).

\section{Other Ubiquitous Scenarios}

Ubiquitous computing supports more than the above two scenarios. For example, combined with sensor technology it allows for what is usually referred to as ambient intelligence applications (see, for instance, http://www.research.philips.com/technologies/syst_soft w/ami/background.html). A typical scenario in this domain is: A person is in the living room, watching $\mathrm{TV}$; the phone rings; as the handset is picked up, the TV is muted; after talking on the phone, the volume on the TV is reset to its previous value. From the information management perspective, these systems may be seen as collaborative agents driven by event-based monitoring. Their goal is information control (and subsequent actions), rather than information services.

Yet another scenario is exemplified by tracking applications, e.g.: A parcel of electronic equipments is prepared for shipping from Japan to USA; the manufacturer puts some context-recording sensors into the parcel to take measurements (temperature, humidity, and magnetic field) and track the position of the goods constantly; if pre-specified thresholds are approached, the sensors notify the need to adjust the conditions; if they are violated, the sensors save the data with the timestamp; the receiver can read the recordings. Such scenarios are already operational in the real world. Their characteristic is the use of mobile sensors and wireless communications, as provided by ubiquitous capabilities. However, from the information management perspective they are rather traditional systems, at least in current usage, collecting distant data to perform centralized control of the application behavior.

\section{User Profiles}

User profiles provide information that may help in achieving intelligent and personalized LBS. They have already attracted much attention, in particular from research in artificial intelligence [1] [5], addressing issues such as acquisition of user profiles, learning from user preferences, use of profiles for better information filtering and decision-making. Different profile-aware filtering algorithms have been implemented in applications such as recommendation systems and web browsing [12] [14]. However, most of the proposals are tailored for some specific computing environment or predefined application (see, e.g., [10]). For example, user profiles are routinely used by Web services, which ask users to fill a predefined form to register to the service. The goal is mainly to send personalized advertisements to registered users. Another visible effect of users profiles is given by Web services that, once they know the country of residence of the user, display their Web pages in the language spoken in that country. For example, entering Switzerland as country of residence results in getting the next Web page in German, although the user may be located in the French-speaking or Italian-speaking parts of Switzerland!

In location-based services, due to the inherent mobility framework, the computing environment is continuously changing, as well as the type and functionality of available data sources. Static approaches are therefore poorly useful. In LBS, both the environment and the user profile may change anytime due to change in user's location, in the social environment (e.g., entering or leaving a meeting), and in the user's activity (e.g., from professional to leisure). Hence, LBS rather have to focus on more generic and dynamic techniques, any time capable of adjusting their services to the current environment and user profile.

For user profile acquisition, it is important to avoid lengthy questionnaires. Experiments have shown that users faced to more than 4 prompts for information from a query system tend to give up using the system [15]. LBS can limit the number of interactions with the users by building upon the matching of the user profile with the knowledge they maintain about the data 
sources. For example, it is obviously useless to ask users which type of cuisine they prefer if the description of restaurants in the data sources does not include the type of cuisine they offer. Usability, selectivity of attributes and frequency of use are relevant criteria in choosing what to look for to make up a user profile. User profiles may also be acquired and enriched by incrementally deriving relevant data from user interaction, and by deriving additional data by reasoning on an ontology.

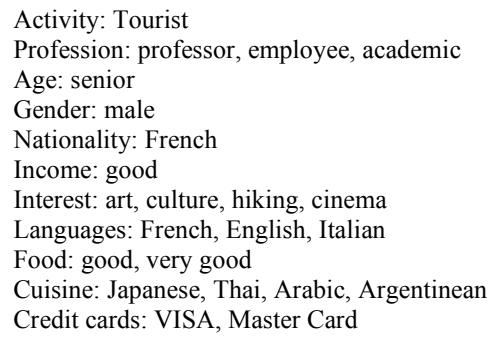

Figure 2. An example profile (a) for Stefano

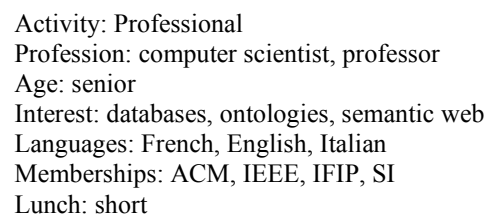

\section{Figure 3. Another example profile (b) for} Stefano

While the same user can have many profiles, at a given point in time, one of these profiles is the one primarily corresponding to the current user's activity and request. Figures 2 and 3 show two possible profiles for the same person, the former relates to his role as a tourist, the latter relates to his role as a computer science professional. This suggests that user profiles may be organized into two parts, a static part and a dynamic part. The static part would store the information that is seen as inherently related to the user, irrespectively of what are his/her current activity and interest, e.g. age and nationality. The dynamic part would contain information closely related to the user's possible activities and requests. In the example profiles for Stefano, the properties 'age', 'gender', 'nationality', 'income' and 'languages' would belong to the static and general part, while 'activity', 'profession', 'interest', 'food', 'cuisine', 'credit cards', 'memberships', and 'lunch' would belong to the dynamic part.

Intelligent LBS are not constrained to using a single profile at a time. For example, a request for information on nearby restaurants for lunch would be best served by considering the current profile, e.g., professional, from which it may be inferred that the planned lunch is a business lunch, extracting the relevant element (lunch, indicating the user preference for a short business lunch rather than a longer one), deriving from a domain ontology some characteristics of business lunches (e.g., the restaurant has to be semi-formal or formal, food quality should be at least good, ambience should be quiet, exotic cuisine styles should get low priority), and finally combine these selection criteria with the ones from the other user profile (namely, cuisine preferences), as a tourist, to suggest an order of preference among the final candidate restaurants (e.g., keep Japanese cuisine in the final choice, to please the user, but give lower priority than traditional cuisine, to conform to the social rules from the ontology).

It is worth noting that even though some attributes are repeated in both profiles, such as 'age' and 'languages', they may lead to different inferences, in the different profiles. A possible organization of user profiles is shown in Fig. 4. It shows, for the same user, two activity-based profiles and one complete profile. The complete profile gathers all data about the user. Some of this data show a mark ( $\mathrm{T}$ for tourist and $\mathrm{P}$ for professional) indicating that the attribute or the attribute value is specific to a given profile (here we assume one profile per activity). For example, 'profession' has one set of values specific to the tourist profile and another set of values specific to the professional profile. 'memberships' shows only one set of values, for the professional profile, but is not seen as specific to this profile (other sets of values may be defined for other profiles). Some data, e.g. 'languages', bears multiple marks, meaning it is shared by the given profiles. Data that is not marked is available for inclusion in any profile (but not necessarily included in all profiles). For example, 'age' is not associated to a specific profile; it is included in both example profiles. 'income' is also not associated to a specific profile, but is included only in the tourist profile. The complete profile is a kind of multi-representation object type, as it holds representations of the user profile for different activities [13]. 


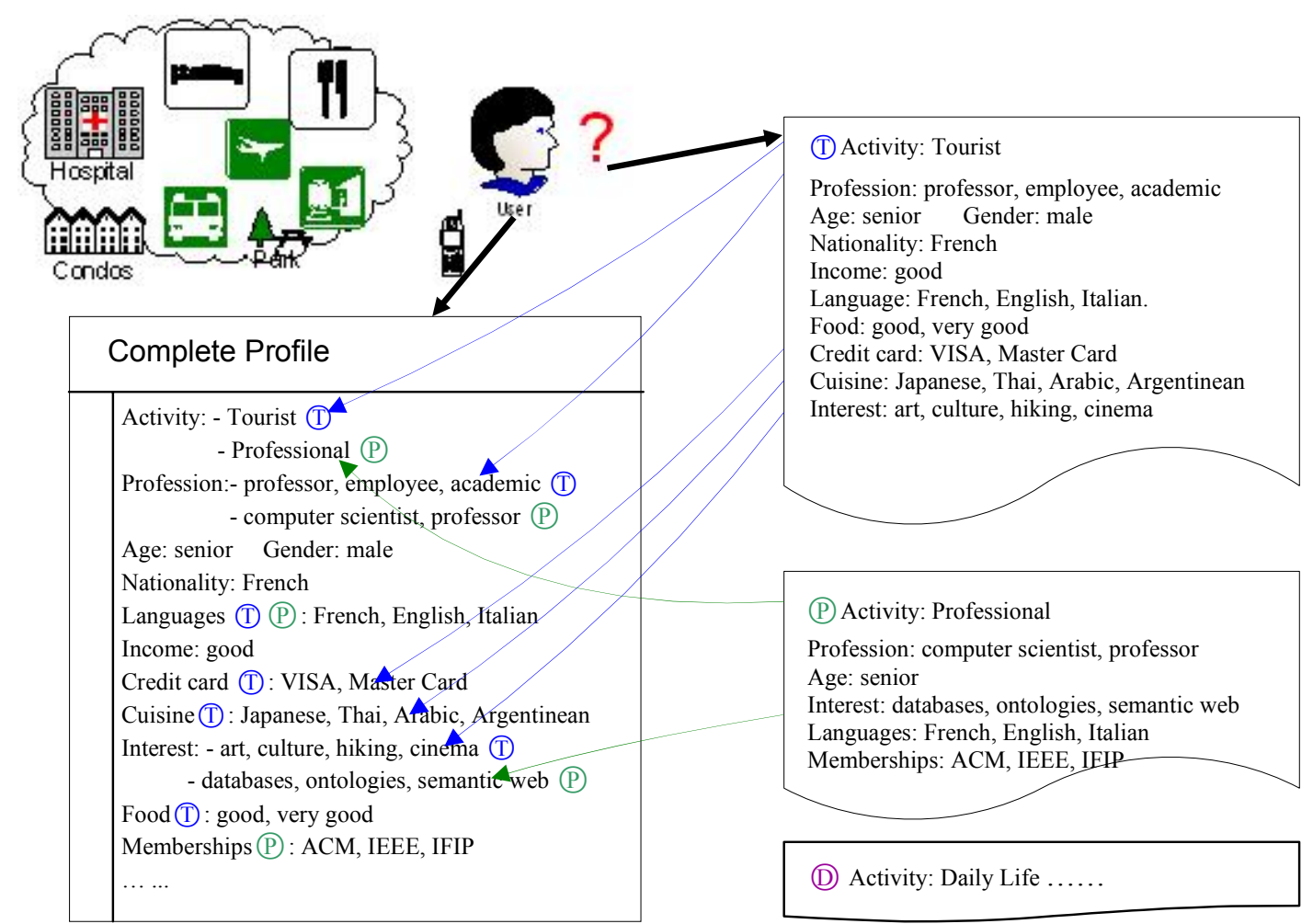

Figure 4. A possible organization of user profiles in LBS

User profiles can be complemented with a personal ontology (i.e., a domain ontology whose domain is this user's concerns and vocabulary). Such an ontology may be of great help in adapting user's profile to local languages, culture, and habits, which the user may not be aware of. For example, a request from a French user to book a table for dinner should be sent to a Swiss data source as a request for a table for the evening meal ("souper"), not a table for "diner", which in Swiss French refers to lunch. Ontologies can also help users to create their own concept hierarchies (including the user profile) for website browsing [7].

More concerns about user profiles include where to store them (e.g., how much can be stored in the user device, and how much in the LBS), and how to maintain them. Notice that acquisition and maintenance of user profiles by the LBS can include reasoning over a number of similar profiles to extract generic knowledge that could be relevant for all similar profiles. For example, the LBS could get clues on a specific user profile by looking at other user profiles in the same clustering group.
User profiles contribute to let LBS properly understand what the user is looking for. In database terms, they are used to rewrite the user query to make it more precise and more specific. For example, the query "where can I buy a souvenir?" can be turned into "give me a nearby department store which sells souvenirs" if the LBS can derive from the user profile that the user is looking for inexpensive souvenirs, more likely to be found in department stores than in specialized shops. Similarly, the query "which movie could I see tonight?" can be enriched with user preferences and be reformulated as "select a movie in English or French or Italian which was rated at least good and is not an action movie".

User profiles can also be used for predicting upcoming behavior of the user, according to existing generic or personal patterns, e.g., alerting user it's time for lunch at about the time the user uses to go for lunch. Finally, they can be used to present retrieved information according to user's order of preference. 


\section{Contexts}

Everything is context-dependent, in particular the meaning of the terms that appear in user's requests or in service and data descriptions. Yet traditional database technology mostly ignores context, providing data services based on a single, implicit context. Fortunately, contexts are now a popular research area, in particular for the database community (cf. these UDM'05 proceedings, where almost every paper deals with contexts).

Contexts in LBS group any information that characterizes the situation of a person, place, or object, as well as the meaning of things at hand, and that can be used to provide more relevant services to the user. LBS contextual information typically includes the user's location, current time, weather, traffic conditions, etc. In several works context includes the user profile. We prefer to separate what is specific to the user (and relates to his/her profile) from what relates to other (contextual) factors that constrain the user but do not depend on him/her. Systems that use contexts are called context-aware systems.

Context can be used as hard or soft criteria in the selection of relevant services [9]. Hard criteria discard the services that do not meet them, while soft criteria order the set of selected services. For example, the user's location can be used to select only the restaurants within a certain distance from the user (hard criterion), or it can be used to sort the selected restaurants according to their distance from the user (soft criterion).

Contextual information has many alternative representations, which makes it difficult to interpret and use. Context providers and context consumers may have different understandings of the same contextual information. Definition of metadata standards is one way to solve the issue, but is unlikely to scale up to all data services that will exist in the future. Another way to go, nowadays very popular, is using ontologies tailored to provide a shared understanding of the concepts used to describe the context and the data services.

As user profiles, contexts in LBS are highly dynamic and cannot be retrieved once for all, but have to be monitored in order to get the up-to-date contextual information.

As for user profiles, the semantic challenge in LBS context management is to determine which contextual information is relevant for the current task. It does not make sense to manage very sophisticated contexts if only a few components are actually useful. Conversely, lower quality services are offered if the appropriate contextual information is missing. Determining what is relevant in a context calls for matching the context with the user profiles on one hand and with the data profiles on the other hand.

A detailed overview of context characteristics and issues may be found elsewhere in this volume [4]. TIP (Tourism Information Provider) system [6], COMPASS (COntext-aware Mobile Personal ASSistant) for mobile tourist applications [9], and the COSS (Context-aware, Ontology-based, Semantic Service discovery) system [3], are excellent examples of context-aware systems, the latter including the use of several domain-specific ontologies: Service type ontology (containing concepts such as shop, restaurant), product ontology (containing concepts such as DVD, vegetarian food), payment ontology (containing concepts such as cash, credit card), and the context ontology (containing concepts such as location, time).

\section{Conclusion}

Ubiquitous computing is the IT revolution of the $21^{\text {st }}$ century. It entails a variety of new approaches to information management and dissemination. The common goal for these approaches is to provide personalized and contextualized information services, contrasting with the anonymous and all-embracing information currently provided by Web services.

In this paper we surveyed different types of services, focusing on promising and innovative solutions such as broadcast-based and location-based services. We further discussed user profiles, the essential contributor to personalization, and highlighted the importance of contextual data, for which the reader can find extensive coverage in the other papers within this same volume.

Discussions with late Prof. Yahiko Kambayashi significantly contributed to the ideas in this paper. We are happy to see our paper in the proceedings of the workshop dedicated to his memory.

\section{References}

[1] Basu, C., Hirsh, H., and Cohen, W. Recommendation as Classification: Using Social and Content-Based Information in Recommendation. In Proceedings of the $\mathrm{Na}$ tional Conference on Artificial Intelligence (AAAI '98) (Madison, Wisconsin, USA, July, 1998), AAAI Press, 1998, 714-720.

[2] Bharat, R., and Minakakis, L. Evolution of Mobile Location-based Services. Communications of the ACM, 46, 12 (Dec. 2003), 61-65.

[3] Broens, T., Pokraev, S., van Sinderen, M., Koolwaaij, J., and Costa, P. D. Context-Aware, Ontology-Based Service Discovery. In Proceedings of the European Symposium on Ambient Intelligence (EUSAI'04) (EindHoven, 
The Netherlands, November, 2004). Springer, 2004, $72-$ 83.

[4] van Bunningen, A.H., Feng, L., and Apers, P.M.G. Context for Ubiquitous Data Management. In this volume.

[5] Chen, L., and Sycara, K. WebMate: A Personal Agent for Browsing and Searching. In Proceedings of the International Conference on Autonomous Agents (Agents'98) (Minneapolis/St. Paul, USA, May, 1998), ACM Press, 1998, 132-139.

[6] Hinze, A., and Voisard, A. Location- and Time-Based Information Delivery in Tourism. In Proceedings of the International Symposium on Advances in Spatial and Temporal Databases (SSTD'03) (Santorini Island, Greece, July, 2003). Springer, 2003, 489-507.

[7] Pretschner, A., and Gauch, S. Ontology Based Personalized Search. In Proceedings of the IEEE International Conference on Tools with Artificial Intelligence (ICTAI 1999) (Chicago, Illinois, USA, November, 1999), IEEE Computer Society, 1999, 391-398.

[8] Schiller, J., and Voisard, A. (Eds.) Location-Based Services. Elsevier Morgan Kaufman, 2004

[9] van Setten, M., Pokraev, S., and Koolwaaij, J. ContextAware Recommendations in the Mobile Tourist Application COMPASS, In Proceedings of the International Conference on Adaptive Hypermedia and Adaptive Web-Based Systems (AH'04) (Eindhoven, The Netherlands, August, 2004). Springer, 2004, 235-244.

[10] Smyth, B., and Cotter, P. A Personalized Television Listings Services. Communications of the ACM, 43, 8 (Aug. 2000), 107-111.

[11] Spiekermann, S. General Aspects of Location-Based Services. In Location-Based Services. Schiller, J., and Voisard, A. (Eds.). Morgan Kaufman, 2004.

[12] Sugiyama, K., Hatano, K., Yoshikawa, M., and Uermura, S. User-Oriented Adaptive Web Information Retrieval Based On Implicit Observation. In Proceedings of the Asia Pacific Web Conference (APWeb'04) (Hangzhou, China, April, 2004), Springer, 2004, 636-643.

[13] Vangenot, C., Parent, C., and Spaccapietra, S. Modeling and manipulating multiple representations of spatial data. In Proceedings of the International Symposium on Spatial Data Handling (SDH 2002), Ottawa, Canada, July 9-12 2002.

[14] Widyantora, D.H., Ioerger, T.R., Yen, J. Learning User Interest Dynamics with a Three-Descriptor Representation. Journal of the American Society of Information Science and Technology (JASIST), 52, 3 (Feb. 2001), 212-225.

[15] Wu, H., Li, M., Zhang, H., and Ma, W. Improving Image Retrieval with Semantic Classification Using Relevance Feedback. In Proceedings of the IFIP TC2/WG2.6 Working Conference on Visual Database Systems (VDB'02) (Brisbane, Australia, May, 2002), Kluwer, 2002, 327-339. 